\title{
Reinnervation of Vastus lateralis is increased significantly in seniors (70-years old) with a lifelong history of high-level exercise
}

\author{
Simone Mosole $(1,2)^{*}$, Katia Rossini (1,2), Helmut Kern (2), Stefan Löfler (2), Hannah \\ Fruhmann (2), Michael Vogelauer (3), Samantha Burggraf (2), Martina Grim-Stieger (3), Ján \\ Cvečka (4), Dušan Hamar (4), Milan Sedliak (4), Nejc Šarabon (5), Amber Pond (6), Donatella \\ Biral (7), Ugo Carraro (1)*, Sandra Zampieri $(1,2)$
}

(1) Laboratory of Translation Myology, Department of Biomedical Sciences, Padova, Italy; (2) Ludwig Boltzmann Institute of Electrical Stimulation and Physical Rehabilitation, Vienna, Austria; (3) Department of Physical Medicine and Rehabilitation, Wilhelminenspital, Vienna, Austria; (4) Faculty of Physical Education and Sport, Comenius University, Bratislava, Slovakia; (5) University of Primorska, Science and Research Centre, Institute for Kinesilogical Research, Koper, Slovenia; (6) Anatomy Department, Southern Illinois University School of Medicine, Carbondale, IL, United States; C.N.R. Institute of Neuroscience, Department of Biomedical Sciences, Padova, Italy

${ }^{*}$ SM and UC contributed equally to the work.

\begin{abstract}
It has long been recognized that histological changes observed in aging muscle suggest that denervation contributes to muscle deterioration and that disuse accelerates the process while running activity, sustained for decades, protects against age-related loss of motor units. Here we show at the histological level that lifelong increased physical activity promotes reinnervation of muscle fibers. In muscle biopsies from 70 -year old men with a lifelong history of high-level physical activity, we observed a considerable increase in fiber-type groupings (almost exclusively of the slow type) in comparison to sedentary seniors, revealing a large population of reinnervated muscle fibers in the sportsmen. Slow-type transformation by reinnervation in senior sportsmen seems to be a clinically relevant mechanism: the muscle biopsies fluctuate from those with scarce fiber-type transformation and groupings to almost fully transformed muscle, going through a process in which isolated fibers co-expressing fast and slow MHCs seems to fill the gaps. Taken together, our results suggest that, beyond the direct effects of aging on the muscle fibers, changes occurring in skeletal muscle tissue appear to be largely, although not solely, a result of sparse denervation. Our data suggest that lifelong exercise allows the body to adapt to the consequences of the age-related denervation and to preserve muscle structure and function by saving otherwise lost muscle fibers through recruitment to different, mainly slow, motor units. These beneficial effects on motoneurons and, subsequently on muscle fibers, serve to maintain size, structure and function of muscle fibers, delaying the functional decline and loss of independence that are commonly seen in late aging.

Trial Registration: ClinicalTrials.gov: NCT01679977
\end{abstract}

Key Words: aging, human skeletal muscle, lifelong physical exercise, senior sportsmen, denervation and reinnervation, fiber-type grouping, training

European Journal Translational Myology - Basic Applied Myology 2013; 23 (4): 205-210

It has long been accepted that histological changes seen in aging muscle suggest that denervation significantly contributes to tissue atrophy [28,30]. Corroborating evidence of a progressive loss of $\alpha$ motoneurons has been described with aging [27] and electrophysiological studies have confirmed a decrease in the number of motor units with some increase in their size, suggesting reinnervation effort [7]. Further evidence supporting rounds of denervation and reinnervation is based on the observation that in young 


\section{Increased reinnervation in aging by longlife high level exercise}

European Journal Translational Myology - Basic Applied Myology 2013; 23 (4): 205-210

humans, fiber types appear randomly distributed across the muscle but become increasingly clustered or grouped together with age [1]. Therefore, it has been proposed that apoptosis of motoneurons in the spinal cord (with subsequent incomplete reinnervation of fibers by surviving motoneurons) contributes to the loss of muscle strength and mass that occurs with age [17].

All of these processes are accompanied by a progressive increase in slow muscle fibers, although the literature provides some contradictions (see recent review [22]). Some of this discrepancy has been dispelled by comparisons of muscle from active and immobile patients: the immobile elderly have a shift toward fast isoform expression, as is common in "unloaded" muscle (e.g., during spaceflight or limb immobilization), whereas muscle wasting is accompanied by a shift toward a fast twitch phenotype [26]. Thus the actual expression pattern of myosin isoforms in the elderly is modulated by complex factors because it depends upon the conflicting influences of both aging and reduced activity tending to shift toward slow and fast isoforms, respectively [6]. To further complicate the situation, conflicting results regarding fast to slow myosin transition arise in endurance training studies using animal models and in clinical trials of humans involving either voluntary exercise or electrical stimulation (directly to muscle or indirectly through nerve stimulation) $[3,4,11,18$, $21,24,26]$. Whether these shifts are under neural control or the direct effect of use/disuse on muscle fibers remains to be clarified.

In the presents study, we analyzed muscle biopsies harvested from the Vastus lateralis of senior (65 to 79 years) amateur sportsmen (i.e., subjects who routinely practice sport activities usually more than three times a week, up to the time of biopsy). In agreements with some previous studies of master athletes [5,29,31], we show that lifelong high-level physical activity considerably increases the percentage of slow-type myofibers and the number of muscle fiber-type groupings. Slow-type transformation by reinnervation in senior sportsmen appears to be a clinically relevant mechanism because, despite the facts that the biopsies from our subjects vary in the degree to which they have undergone slow-type transformation and that numerous factors can affect fiber type transition, the analyses of our data demonstrate that the senior sportsmen have a significantly greater level of slow type fiber groupings, demonstrating that their muscle has undergone significant reinnervation. Indeed, in recent meetings, we have reported that muscle properties of these senior amateur sportsmen are more similar to those of active young men than to those of sedentary seniors $[13,33]$. Thus our studies support the concept that lifelong high-level exercise has a beneficial effect on the motoneurons and, through them, on the muscle fibers, resulting in maintainance of muscle size, structure and function, thereby delaying the functional decline and loss of independence that are commonly seen in aging adults.

\section{Materials and Methods}

All subjects recruited for the study were volunteers who received detailed information and all signed an informed consent. Approval from the national committee for medical ethics was obtained before study onset (EK08-102-0608). Groups of young men $(n=16)$, seniors with normal life style (sedentary, $n=16)$ and seniors with a lifelong history of high-level

Table 1. Small angular muscle fibers in young men and in septuagenarians either sedentary or sportsmen

Myofiber diameter

\begin{tabular}{lllll}
\hline & & $<25 \mu \mathrm{m}$ \\
& ANOVA & $\%$ & ANOVA
\end{tabular}

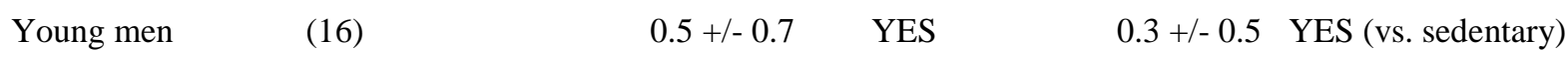

Seniors

\begin{tabular}{llllll}
\hline Sedentary & $(16)$ & $6.9+/-3.6$ & YES & $3.0+/-2.0$ & YES (vs Sportsmen) \\
Sportsmen & $(16)$ & $1.0+/-1.7$ & NO & $0.2+/-0.3$ & NO (vs. Youngs)
\end{tabular}

YES or NO, significance of ANOVA test. 
Increased reinnervation in aging by longlife high level exercise

European Journal Translational Myology - Basic Applied Myology 2013; 23 (4): 205-210

Table 2. Fiber-type groupings in young men and in septuagenarians either sedentary or sportsmen

Fiber-type groupings

(as \% of central fibers in clustered areas vs. total fibers)

\begin{tabular}{|c|c|c|c|c|c|}
\hline Subjects & (size) & Fast & ANOVA & Slow & ANOVA \\
\hline Young men & (8) & $1.1+/-2.2$ & NO & $<0.1+/-0.1$ & NO (vs. sedentary) \\
\hline
\end{tabular}

Seniors

\begin{tabular}{llllll}
\hline Sedentary & $(8)$ & $3.6+/-5.1$ & NO & $0.5+/-0.6$ & YES (vs. Sportsmen) \\
Sportsmen & $(8)$ & $0.1+/-0.1$ & NO & $8.4+/-7.9$ & YES (vs. Young men)
\end{tabular}

YES or NO, significance of ANOVA test.

recreational sport activities $(n=16)$ were enrolled. All subjects were healthy and declared not to have any specific mobility impairment or disease. Upon enrollment in the study, needle muscle biopsies were harvested through a small skin incision $(6 \mathrm{~mm})$ from the right and left Vastus lateralis muscles of each patient and then frozen for light microscopy as described [11].

Light microscopy and quantitative histological analyses. Serial cryosections $(8 \mu \mathrm{m})$ from frozen muscle biopsies were mounted on polysine ${ }^{\mathrm{TM}}$ glass slides, air-dried and stained either with Hematoxylin and Eosin (H\&E) or using conventional techniques for myofibrillar ATPases to evaluate muscle fiber types [23]. In the latter method, slow-type muscle fibers are dark while the fast-type fibers are lightly stained following preincubation at $\mathrm{pH} 4.35$. The reverse is true after preincubation at $\mathrm{pH} 9.4$.

Morphometric analyses of the fiber diameter and of the fiber type distribution were performed on cryosections using Scion Image for Windows version Beta 4.0.2 (2000 Scion Corporation) as previously described [913,23].

Statistical analysis. ANOVA tests were performed with statistics algorithms of Origin ${ }^{\mathrm{TM}}$, OriginLab Corporation, USA. The level of statistical significance was set at $\mathrm{p}<0.05$.

\section{Results and Discussion}

From our previous studies on skeletal muscle biopsies of paraplegic patients we know that muscle disuse resulting from decades of years of denervation (after upper motor neuron lesion) induces at most a $50 \%$ decrease in size (i.e., from a myofiber diameter of approximately $70 \mu \mathrm{m}$ to $35 \mu \mathrm{m}$ ) [12], while lower motor neuron denervated skeletal muscle (one year after denervation) shows muscle fibers with a diameter less than $30 \mu \mathrm{m}$ [2,9-11]. Based upon these findings, we are confident in defining those muscle fibers having a diameter smaller than $30 \mu \mathrm{m}$ as denervated. This interpretation is strengthened by the fact that several small myofibers have angular aspects [33]. In the biopsies analyzed here, small angular muscle fibers have the size and the morphology of denervated muscle fibers and they are more frequent in sedentary septuagenarians than in young men and septuagenarians with a lifelong history of high-level exercise (Table 1). Muscle fibers with a diameter less than $30 \mu \mathrm{m}$ are seldom observed $(<0.5 \%)$ in the muscle biopsies of young men, while biopsies harvested from the sedentary seniors contain the highest percentage $(6.9 \%)$ of denervated muscle fibers among the three groups (Table 1). When muscle fibers with diameters less than $25 \mu \mathrm{m}$ are counted the percentages decrease by approximately $50 \%$ for each group, however, the sedentary seniors still maintain the highst values. ANOVA tests on these data confirm that the higher percentages of small angular fibers in sedentary seniors relative to both young subjects and senior sportsmen are statistically significant. This is not the case when young subjects and senior sportsmen are compared.

Analyses of fiber-type groupings demonstrate that, although not statistically significant, the percentage of fast fiber types is markedly higher in the sedentary seniors than in either the senior sportsmen or the young men. The percentage of slow type fibers, however, is significantly higher in the senior men (both sedentary and sportsmen) than in the younger men. Most interestingly, the percentage of slow-type fibers in the senior sportsmen is signficantly higher than in the sedentary seniors (Table 2). 
Increased reinnervation in aging by longlife high level exercise

European Journal Translational Myology - Basic Applied Myology 2013; 23 (4): 205-210

Fiber-type grouping is identified on the basis that one myofiber is completely surrounded by fibers of the same phenotype. Because two or more slow type fibers were not always easily distinguished one from another in alkaline-resistant ATPase specimens, we confirmed our fiber border delineations with the less ambiguous method of acid resistant ATPase staining of specimens following preincubation at $\mathrm{pH}$ 4.35. In figure 1 some examples of ATPase staining of muscle biopsies harvested from high-level recreational sportsmen are shown, beginning with one which contains a one-toone proportion of slow-to-fast fibers (as in normal adult muscles) and escalating to a sample in which

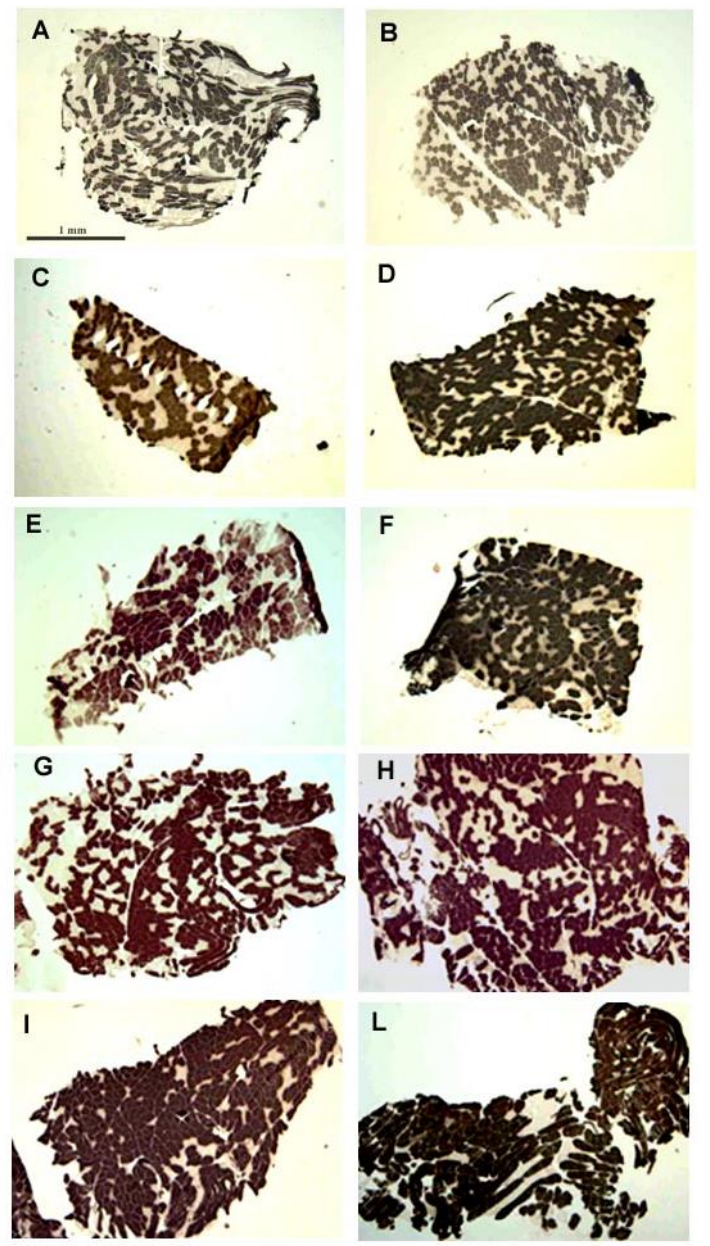

Fig. 1 Fiber type distribution by ATPase staining (pH 4.35) in 70-year sportsmen shows a high occurrence of slow type fibers (dark stained myofibers). Biopsies are ordered from panel A to panel $\mathrm{L}$ according to their increasing percentage of slow fibers. The majority has around $70 \%$ of slow type, ranging from $51 \%$ (panel A), to $92 \%$ (panel L). See also Table 3. All panels are at the same magnification, bar $=1 \mathrm{~mm}$. almost all the muscle biopsy is covered by very large slow fiber-type groupings.

Table 2 shows that some fast fiber-type groupings were present in the biopsies harvested from sedentary seniors: the central fibers characterizing fast fiber-type groupings were $3.6 \%$ of the total muscle fibers, while those of slow-type were around $0.5 \%$. Even more evident is the fact that, in the biopsies harvested from senior sportsmen, the slow type fibers are grouped in larger areas (mean $8.4 \%$, see Table 2), almost reaching the $92 \%$ in the extreme cases (Table 3 ).

It has long been recognized that the histological changes seen in aging muscle suggest that denervation significantly contributes to muscle decay $[8,25,28]$ and that immobility accelerates the deterioration process [6], while running activity sustained for decades (as that performed by master athletes) protects against the age-related loss of motor units $[16,19,20]$ and, thereby, protects lean muscle mass [32]. However, the degree to which denervation causes muscle fiber transformation and loss of myofibers is an open issue in humans, since reinnervation events may compensate long-term for motor neuron loss in spinal cord and/or axonal abnormalities in peripheral nerves $[1,7,14,15]$.

In the present study we used histochemical ATPase methods to analyze muscle biopsies harvested from septuagenarian sportsmen and compared their relative amount of: 1. small angular myofibers (denervated muscle fibers), 2. fast and slow muscle fibers (muscle plasticity), and 3. central muscle fibers of fiber-type clusters (reinnervated muscle fibers) with those in muscle biopsies of sedentary septuagenarians and young men. The main results are: 1 . biopsies from young men seldom contain denervated and

Table 3. Slow fibers and fiber-type groupings in Vastus lateralis of 70-year sportsmen

$\begin{array}{ccc}\text { Panels } & \begin{array}{c}\text { Slow fibers } \\ (\%)\end{array} & \begin{array}{c}\text { Slow groupings } \\ (\#)\end{array} \\ \text { A } & 51 & 2 \\ \text { B } & 68 & 6 \\ \text { C } & 69 & 3 \\ \text { D } & 70 & 19 \\ \text { E } & 71 & 6 \\ \text { F } & 75 & 4 \\ \text { G } & 76 & 18 \\ \text { H } & 81 & 23 \\ \text { I } & 85 & >23 \\ \text { L } & 92 & >23\end{array}$




\section{Increased reinnervation in aging by longlife high level exercise}

European Journal Translational Myology - Basic Applied Myology 2013; 23 (4): 205-210

reinnervated muscle fibers or transforming myofibers; 2. biopsies from sedentary seniors contain both denervated and a few reinnervated clustered myofibers of the fast type; and 3. senior sportsmen present with a larger percentage of slow myofibers, up to $90 \%$, which appear clustered in slow fiber-type groupings. Our data suggest that slow-type transformation by reinnervation in senior sportsmen is a clinically relevant mechanism despite the facts: 1) that subject biopsies vary from those with scarce fiber-type transformation and groupings to those with almost fully transformed muscles in which isolated fibers co-expressing fast and slow MHCs fill in the gaps (Mosole et al., manuscript in preparation); and 2) there are potential confounding factors such as the sampling of a heterogeneous muscle, individual genetic backgrounds, difference in kind and extent of the high level activities.Indeed, in recent meetings we reported that the muscle properties of this group of senior sportsmen are more similar to that of active young men than to those of sedentary seniors. Specifically, the results indicate that relative to their sedentary cohorts, senior sportsmen have greater muscle maximal isometric force and function and better preserved muscle morphology and ultrastructure $[13,33]$.

Taken together our results suggest that, beyond the direct effects of aging on the muscle fibers, changes occurring in skeletal muscle tissue appear to be largely, although not solely, a result of sparse incremental denervation. In senior sportsmen the increase in slow clustered fiber percentage is conceivably the result of the positive effect of lifelong physical activity on the motoneuron pool, which has spared the slow motoneurons from age related lesion/death, increasing the chance that peripheral reinnervation occurs due to sprouting of slow axons. Lifelong exercise seems to allow the body to adapt to the consequences of agerelated denervation and to preserve muscle structure and function by saving otherwise lost muscle fibers through recruitment to different, mainly slow, motor units. Thus, regular physical activity is a good strategy to attenuate muscle functional decline and ultrastructural abnormalities associated with aging. Certainly other mechanisms contribute to lifelong muscle health, however, our present data support the concept that lifelong high-level exercise has a beneficial effect on the motoneurons and, through them, on muscle fibers, thereby maintaining size, structure and function and thus delaying age-related functional decline and loss of independence that are commonly seen in late aging.

\section{Acknowledgements}

The authors would like to acknowledge the support of: (1) the European Regional Development Fund for funding the Cross Border Cooperation Programme Slovakia - Austria 2007 - 2013 (Interreg-IVa), project Mobilität im Alter, MOBIL, N_00033 (partners:
Ludwig Boltzmann Institute of Electrical Stimulation and Physical Rehabilitation, Austria, Center for Medical Physics and Biomedical Engineering, Medical University of Vienna, Austria and Faculty of Physical Education and Sports, Comenius University in Bratislava, Slovakia); (2) the Austrian national cofinancing of the Austrian Federal Ministry of Science and Research, and (3) MIUR Italy, Funds ex-60\% to UC, Laboratory of Translation Myology, Department of Biomedical Sciences, Padova, Italy.

\section{Corresponding Author}

Sandra Zampieri, PhD. Laboratory of Translation Myology, Department of Biomedical Sciences, Padova, Italy; e-mail: sanzamp@unipd.it

\section{References}

[1] Andersen JL. Muscle fibre type adaptation in the elderly human muscle. Scand J Med Sci Sports 2003;13:40-47. doi: 10.1034/j.16000838.2003.00299.x.

[2] Boncompagni S, Kern H, Rossini K, Hofer C, Mayr W, Carraro U, Protasi F. Structural differentiation of skeletal muscle fibers in the absence of innervation in humans. Proc Natl Acad Sci USA 2007;104:19339-19344. Epub 2007 Nov 27.

[3] Carraro U. Modulation of trophism and fiber type expression of denervated muscle by different patterns of electrical stimulation. Basic Appl Myol 2002;12:263-273.

[4] Carraro U, Catani C, Belluco S, Cantini M, Marchioro L. Slow-like electrostimulation switches on slow myosin in denervated fast muscle. Exp Neurol 1986;94:537-553.

[5] Coggan AR, Spina RJ, Rogers MA, King DS, Brown M, Nemeth PM, Holloszy JO. Histochemical and enzymatic characteristics of skeletal muscle in master athletes. J Appl Physiol 1990;68:1896-1901. doi: 10.1063/1.346579.

[6] D’Antona G, Pellegrino MA, Adami R, Rossi R, Carlizzi CN, Canepari M, Saltin B, Bottinelli R. The effect of ageing and immobilization on structure and function of human skeletal muscle fibres. J Physiol (Lond.) 2003;552:499-511.

[7] Doherty TJ, Vandervoort AA, Taylor AW, Brown WF. Effects of motor unit losses on strength in older men and women. J Appl Physiol 1993;74: 868-874. doi: 10.1063/1.354879.

[8] Gutmann E, Hanzlikova V. Motor unit in old age. Nature 1966;209:921-922.

[9] Kern H, Boncompagni S, Rossini K, Mayr W, Fanò G, Zanin ME, Podhorska-Okolow M, Protasi F, Carraro U. Long-term denervation in humans causes degeneration of both contractile and excitation-contraction coupling apparatus that can be reversed by functional electrical stimulation (FES). A role for myofiber 
Increased reinnervation in aging by longlife high level exercise

European Journal Translational Myology - Basic Applied Myology 2013; 23 (4): 205-210

regeneration? J Neuropath Exp Neurol 2004;63:919-931.

[10] Kern H, Carraro U, Adami N, Hofer C, Loefler S, Vogelauer M, Mayr W, Rupp R, Zampieri S. One year of home-based daily FES in complete lower motor neuron paraplegia: recovery of tetanic contractility drives the structural improvements of denervated muscle. Neurol Res 2010;32:5-12. doi: 10.1179/174313209X385644.

[11] Kern H, Carraro U, Adami N, Biral D, Hofer C, Forstner C, Mödlin M, Vogelauer M, Pond A, Boncompagni S, Paolini C, Mayr W, Protasi F, Zampieri S. Home-based functional electrical stimulation rescues permanently denervated muscles in paraplegic patients with complete lower motor neuron lesion. Neurorehabil Neural Repair 2010;24:709-721.

[12] Kern H, Hofer C, Mödlin M, Mayr W, Vindigni V, Zampieri S, Boncompagni S, Protasi F, Carraro U. Stable muscle atrophy in long-term paraplegics with complete upper motor neuron lesion from 3- to 20-year SCI. Spinal Cord 2008;46:293-304.

[13] Kern H, Loefler S, Burggraf S, Fruhmann H, Cvecka J, Sedliak M, Barberi L, De Rossi M, Musarò A, Carraro U, Mosole S, Zampieri S. Electrical stimulation counteracts muscle atrophy associated with aging in humans. European Journal Translational Myology - Basic Applied Myology 2013;23:105-108.

[14] Larsson L. Motor units: remodeling in aged animals. J Gerontol A Biol Sci Med Sci 1995:50:91-95.

[15] Lexell J, Downham DY. The occurrence of fibertype grouping in healthy human muscle: a quantitative study of cross-sections of whole vastus lateralis from men between 15 and 83 years. Acta Neuropathol (Berl) 1991;81:377-381.

[16] Leyk D, Rüther T, Wunderlich M, Sievert A, Essfeld D, Witzki A, Erley O, Küchmeister G, Piekarski C, Löllgen H. Physical performance in middle age and old age: good news for our sedentary and aging society. Dtsch Arztebl Int 2010;107:809-816.

[17] Luff AR. Age-associated changes in the innervation of muscle fibers and changes in the mechanical properties of motor units. Ann N Y Acad Sci 1998;854:92-101.

[18] Mayne CN, Mokrusch T, Jarvis JC, Gilroy SJ, Salmons S. Stimulation-induced expression of slow muscle myosin in a fast muscle of the rat. Evidence of an unrestricted adaptive capacity. FEBS Lett 1993;327:297-300.

[19] McNeil CJ, Doherty TJ, Stashuk DW, Rice CL. Motor unit number estimates in the tibialis anterior muscle of young, old, and very old men. Muscle Nerve 2005;31:461-467.
[20] Meltzer DE. Age dependence of olympic weightlifting ability. Med Sci Sports Exerc 1994;26:1053-1067.

[21] Midrio M. The denervated muscle: facts and hypotheses. A historical review. Eur J Appl Physiol 2006;98:1-21. Epub 2006 Aug 3.

[22] Mitchell WK, Williams J, Atherton P, Larvin M, Lund J, Narici M. Sarcopenia, dynapenia, and the impact of advancing age on human skeletal muscle size and strength; a quantitative review. Front Physiol 2012;3:260. doi: 10.3389/fphys.2012.00260.

[23] Rossini K, Zanin ME, Podhorska-Okolow M, Carraro U. To stage and quantify regenerative myogenesis in human long-term permanent denervated muscle. Basic Appl Myol 2002;12:277-286.

[24] Salmons S. Exercise, stimulation and type transformation of skeletal muscle. Int $\mathbf{J}$ Sports Med 1994;15:136-141. Review.

[25] Scelsi R, Marchetti C, Poggi P. Histochemical and ultrastructural aspects of $\mathrm{m}$. vastus lateralis in sedentary old people (aged 65-89 years). Acta Neuropathol 1980;51:99-105.

[26] Schiaffino S, Reggiani C. Fiber types in mammalian skeletal muscles. Physiol Rev 2011;91:1447-1531.

doi: 10.1152/physrev.00031.2010.

[27] Tomlinson BE, Irving D. The numbers of limb motor neurons in the human lumbosacral cord throughout life. J Neurol Sci 1977;34:213-219.

[28] Tomlinson BE, Walton JN, Rebeiz JJ. The effects of ageing and of cachexia upon skeletal muscle. A histopathological study. J Neurol Sci 1969;9:321-346.

[29] Trappe S. Master athletes. Int J Sport Nutr Exerc Metab 2001;11Suppl:S196-207.

[30] Urbanchek MG, Picken EB, Kalliainen LK, Kuzon WM Jr. Specific force deficit in skeletal muscles of old rats is partially explained by the existence of denervated muscle fibers. J Gerontol A Biol Sci Med Sci 2001;56:B191-B197.

[31] Wright VJ, Perricelli BC. Age-related rates of decline in performance among elite senior athletes. Am J Sports Med 2008;36:443-450. Epub 2007 Nov 30

[32] Wroblewski AP, Amati F, Smiley MA, Goodpaster B, Wright V. Chronic exercise preserves lean muscle mass in masters athletes. Phys Sportsmed 2011;39:172-178. doi: 10.3810/psm.2011.09.1933.

[33] Zampieri S, Rossini K, Carraro U, Kern H. Morphometry of skeletal muscle in sedentary elderly and senior sportsmen. European Journal Translational Myology - Basic Applied Myology 2012;22:13. 